\title{
Modelo de requisitos e componentes técnicos para a formação e gerência de redes de cooperação entre empresas da construção civil
}

\author{
Technician components and requirements model for the \\ formation and management of cooperation networks \\ among civil construction companies
}

\author{
Flávia Vancim Frachone Neves ${ }^{1}$ \\ Fábio Müller Guerrini²
}

\begin{abstract}
Resumo: A indústria da Construção Civil possui um modo de operação semelhante ao das organizações virtuais, mas de forma não estruturada, deixando de proporcionar vantagens competitivas. Para tanto, o artigo propõe um Modelo de Requisitos e Componentes Técnicos, baseado na metodologia EKD (Enterprise Knwoledge Development), para auxiliar a formação e gerência de redes entre empresas da construção civil. Para a elaboração do modelo proposto, foi conduzida uma análise dos projetos internacionais mais relevantes no setor da construção além de estudo de casos contendo sete obras a fim de detectar seus objetivos e pontos falhos a serem reestruturados e adaptados para a indústria da construção civil brasileira. Não obstante, o modelo proposto baseou-se em uma adaptação das melhores práticas de uma arquitetura de referência para redes. Como resultado, a proposição viabiliza uma visão clara dos requisitos necessários, explora a necessidade de intensa comunicação e troca de informações, além de contribuir com pesquisas futuras no que tange a um futuro desenvolvimento de sistema de informação direcionado para este segmento econômico.
\end{abstract}

Palavras-chave: EKD. Modelo de requisitos e componentes técnicos. Redes de cooperação. Gerenciamento na construção civil.

\begin{abstract}
The operational method of civil construction can be analyzed by the outlook of virtual organizations, however not yet structuralized, hence not providing competitive advantages. Thus, this article considers a Technical Components and Requirements Model, based on EKD methodology (Enterprise Knowledge Development), to assist in the formation and management of networks in civil construction companies. For the elaboration of the considered model, an analysis of the most important international construction projects was conducted, in addition to a case study from seven construction projects in order to detect their goals and defective points, to then be reorganized and adapted to the Brazilian civil construction industry. Moreover, the considered model was also based on an adaptation of reference architecture for civil construction networks. As a result, the proposal makes possible, to the stakeholders, a clear view of the necessary requirements, exploring the necessity of intense communication and exchange of information, besides contributing to future research works regarding a future development of an information system directed for this economic segment.
\end{abstract}

Keywords: EKD. Technician components and requirements model. Cooperation networks. Civil construction management.

\section{Introdução}

A indústria da construção civil não possui uma arquitetura de referência genérica adequada às suas necessidades para apoiar a formação e gerência coordenada de redes de cooperação, bem como para auxiliar o correto levantamento de requisitos para implementar um futuro sistema de informação que supra suas necessidades específicas.
Para Lima, Stephens e Böhms (2003), a construção civil ocupa posição estratégica na geração de empregos e começa a demonstrar contínuo esforço para melhoria do processo com a utilização de parcerias. Em contrapartida, Cheng e Li (2002), Chan et al. (2004) evidenciam o baixo grau de inovação, comunicação, confiança e cooperatividade coordenada. Segundo

\footnotetext{
Escola de Engenharia de São Carlos - EESC, Universidade de São Paulo - USP, Av. Trabalhador São-carlense, 400, CEP 13566-590, São Carlos - SP, Brasil, E-mails: frachone@sc.usp.br; guerrini@sc.usp.br
} 
Zarli e Richaud (1999), a cultura é conservadora e a maioria dos trabalhos são projetos únicos.

Parte dos problemas no setor da construção, de acordo com Wong, Cheung e Ho (2005), ocorre devido às barreiras no aprendizado e mudança organizacional. Isto se torna um entrave ao crescimento. Segundo Belusi e Arcangeli (1998), Matthews (2004), Jones (2005) e Ploetner e Ehret (2006), a competição global associada às rápidas mudanças tecnológicas e à crescente diversidade de produtos tem levado as empresas a implementar novas tecnologias, práticas de gerenciamento, procedimentos e modelos de processos e desenvolvimento de habilidades e competências de seus colaboradores. Neste cenário, as redes de cooperação entre empresas têm gerado ganhos de escala e escopo para as empresas envolvidas.

\subsection{Redes de cooperação}

As redes de cooperação, de acordo com Black, Akintoye e Fitzgerald (2000), Williams (2002), Rycroft e Kash (2004), Buhman (2005), Chien e Peng (2005), Hyder e Eriksson (2005) e Manring e Moore (2006), compõem uma inter-relação empresarial, integrando conhecimentos e habilidades específicas, para executar projetos de interesse comum e atingir objetivos estratégicos competitivos.

Segundo Pfohl e Buse (2000), apesar de relacionarem -se economicamente, são legalmente independentes. Segundo Baldwin e Carter (1999), Gill e Butler (2003), Fitzpatrick e Dilullo (2005), Ho (2006) e Rese (2006), a constituição de uma rede viabiliza benefícios dentre os quais: estipular estratégias conjuntas, preservar a individualidade de cada empresa, possibilitar o marketing compartilhado, reduzir custos de produção e riscos de investimentos, intensificar a comunicação e acesso à informação, conectar habilidades complementares, ampliar a escala produtiva e mercado, facilitar o acesso ao crédito e à capacitação gerencial.

As organizações virtuais são um mecanismo para viabilizar as redes. Esta modalidade de rede, de acordo com Franke (2003), Knouse (2004) e Travica (2005), envolve parceiros geograficamente dispersos que partilham recursos e propósitos, por período determinado, em função de oportunidades e sem compromissos de contato após o término dos episódios.

Segundo Kelley (2001), Breu e Hemingway (2004) e Stowell (2005) o foco das redes é implementar estratégias flexíveis orientadas para o negócio com o apoio da tecnologia de informação, estabelecendo redes de comunicação. Segundo Torkkeli e Tuominem (2002), as redes induzem à redefinição dos níveis de autonomia de cada componente da rede, o que determina a reestruturação de funções e responsabilidades nos centros de trabalho da empresa.
Analisando a infraestrutura necessária para o desenvolvimento de uma organização virtual, é importante, de acordo com Camarinha-Matos e Afsarmanesh (1999), considerar as fases do seu ciclo de vida.

O ciclo de vida de uma organização virtual corresponde às fases de: criação (fase inicial de seleção de parceiros; negociação de contrato; definição de níveis de acesso à informação, de procedimentos conjuntos e configuração); operação (inicia-se com a execução dos processos do negócio a fim de atingir as metas estabelecidas); evolução (ocorre durante a operação, principalmente quando for necessária a substituição ou adição de um parceiro); e dissolução (quando o projeto é finalizado de acordo com os requerimentos inicialmente definidos).

O sucesso da rede relaciona-se ao comportamento não oportunista de cada membro. Conforme Spekman e Carraway (2006), deve haver confiança para que a parceria permaneça. $\mathrm{O}$ desacordo no estabelecimento das metas, entre os parceiros, desencadeia conflitos e instabilidade na rede. Para Rahman e Kumaraswamy (2004), os riscos de instabilidade da rede podem ser transferidos, compartilhados, gerenciados ou minimizados, mas não devem ser ignorados. A confiança, conforme Tomkins (2001), baseia-se na crença de que uma parte possui o compromisso com a outra de não agir contra os interesses em comum.

\subsection{Possibilidades de aplicação do conceito de redes de cooperação no setor da construção civil}

Segundo Rezgui, Zarli e Wilson (2000), Wilson et al. (2001), a construção civil já adota o modo de operação de rede, mas ainda de forma não coordenada. A parceria proporciona um ambiente de múltiplas atividades, modificando e suplementando as barreiras tradicionais entre companhias independentes. De acordo com Black, Akintoye e Fitzgerald (2000), a parceria está começando a ser utilizada nos projetos de construção, envolvendo as partes em um trabalho conjunto, em um ambiente de confiança. Com isto, há diminuição de risco de custos de atrasos e aumento de oportunidade para inovação, devido à comunicação e confiança entre os parceiros envolvidos.

Segundo Glacola e Sheedy (2002), as partes envolvidas na parceria da construção discutem previamente os procedimentos específicos do projeto. Após esta fase, criam-s as equipes de cooperação e desenvolvimento do relacionamento de negócio. Adicionalmente, é necessário que todos os parceiros compreendam claramente as metas.

Entretanto, as parcerias de sucesso não ocorrem naturalmente. Segundo Wong, Cheung e Ho (2005), para desenvolver relações de confiança, são necessários igualdade ou benefícios compartilhados, comunicação 
efetiva e gerenciamento competente. Cheng e $\mathrm{Li}$ (2002) afirmam que há priorização de alguns fatores críticos, entre os quais: suporte da alta gerência, confiança mútua, comunicação aberta e coordenação efetiva. Rahman e Kumaraswamy (2005) abordam que o sucesso dos projetos de construção depende de múltiplos fatores, tais como: recursos humanos, materiais, infraestrutura, procedimentos, gerenciamento.

Conforme Holmen, Pedersen e Torvatn (2005), os obstáculos do setor da construção para a cooperação podem ser: o suporte da tecnologia de informação e comunicação deve lidar com fragmentações impostas pelo setor em função da troca de informação e comunicação necessitar de melhoramentos; as interações entre atores não são bem coordenadas; o gerenciamento do suporte da tecnologia de informação e comunicação ainda é realizado de forma tradicional; a documentação dos projetos apresenta redundância e falta de estruturação. Contudo, segundo Stowell (2005), as corporações virtuais de sucesso focam-se principalmente em construir relacionamentos para aumentar a confiança e desenvolver visões compartilhadas.

\subsection{Metodologia Enterprise Knowledge Development - EKD para modelagem organizacional sob o recorte analítico de redes}

A modelagem organizacional, de acordo com Mertins e Jochem (2005), é utilizada tanto como técnica para representar e entender a estrutura e comportamento das organizações quanto como técnica para analisar processos de negócio e, em muitos casos, como suporte técnico para reengenharia de processos de negócios. Em uma organização, se podem modelar e integrar: pedidos dos clientes; produtos manufaturados; processos do negócio; recursos técnicos, informativos e humanos; decisões organizacionais e propriedades de custo e tempo.

Os modelos são guias de referência que viabilizam gerenciar a complexidade dos sistemas produtivos, facilitando a compreensão do funcionamento organizacional, além de disponibilizar uma documentação para aumentar seu autoconhecimento, possibilitando, consequentemente, o melhoramento contínuo de seus processos.

Na manufatura, notam-se avanços significativos a partir da modelagem de arquiteturas de referência, tais como: CIMOSA (Arquitetura de Sistema Aberto para Manufatura Integrada de Computador), que provê guias e linguagem avançada para modelagem de funções, informações, recursos e aspectos organizacionais (VERNADAT, 1996; KOSANKE; VERNADAT; ZELM, 1999); PERA (Arquitetura de Referência de Empresa Purdue), que é uma metodologia detalhada que quebra o ciclo de vida das empresas em fases, sendo cada uma representada por diferentes diagramas (WILLIAMS, 1997); GERAM (Arquitetura e Metodologia de Referência de Empresa Genérica), que é uma generalização da CIMOSA.

A modelagem organizacional baseada na metodologia EKD (Enterprise Knowledge Development) visa o compartilhamento de recursos e competências para aumentar os ganhos em critérios competitivos.

Segundo Bubenko Jr., Brash e Stirna (1998), ela facilita a aprendizagem e comunicação organizacional; desenvolve uma descrição estruturada do negócio para que os analistas da organização possam discutir e determinar mais claramente os objetivos e requisitos dos sistemas; e produz um documento (chamado repositório de conhecimento). Esse documento pode ser utilizado para raciocinar sobre o negócio, discutir mudanças e componentes do sistema de informação, traçar a cadeia de componentes e decisões que possibilitam diversas interpretações do sistema de informação. Ademais, reflete uma coleção de percepções do mundo real, possibilitando, aos participantes, entrarem em contato com questões referentes às suas respectivas funções no negócio, da mesma forma que passam a visualizar o impacto de suas tomadas de decisões.

É uma metodologia que proporciona aos envolvidos no projeto uma óptica mais analítica da organização e seus componentes como um todo, por meio da modelagem organizacional. O EKD é composto pelos modelos de objetivos, regras de negócio, processos de negócio, requisitos e componentes técnicos, atores e recursos e conceitos, conforme demonstra a Figura 1.

O Modelo de Requisitos e Componentes Técnicos, em específico, determina as estruturas e propriedades que deverão compor um futuro sistema de informação. Da mesma forma permite explicitar o potencial da tecnologia de informação para melhoria do processo de negócio. Os componentes presentes no Modelo de Componentes e Requisitos Técnicos, de acordo com Bubenko Jr., Brash e Stirna (1998), são: objetivos do sistema de informação (propriedades mensuráveis ou não mensuráveis, focos, visões, ou direções); requisitos do sistema de informação (requisitos a serem designados para propriedades do sistema de informação, divididos em duas partes: requisito funcional (propriedade funcional do sistema de informação) e requisito não funcional (restrições políticas, de operações, econômicas, de segurança das informações etc.)

\subsection{Projetos internacionais para cooperação entre empresas no setor da construção civil}

A Europa desenvolveu vários projetos para a cooperação na construção civil agrupados em um 


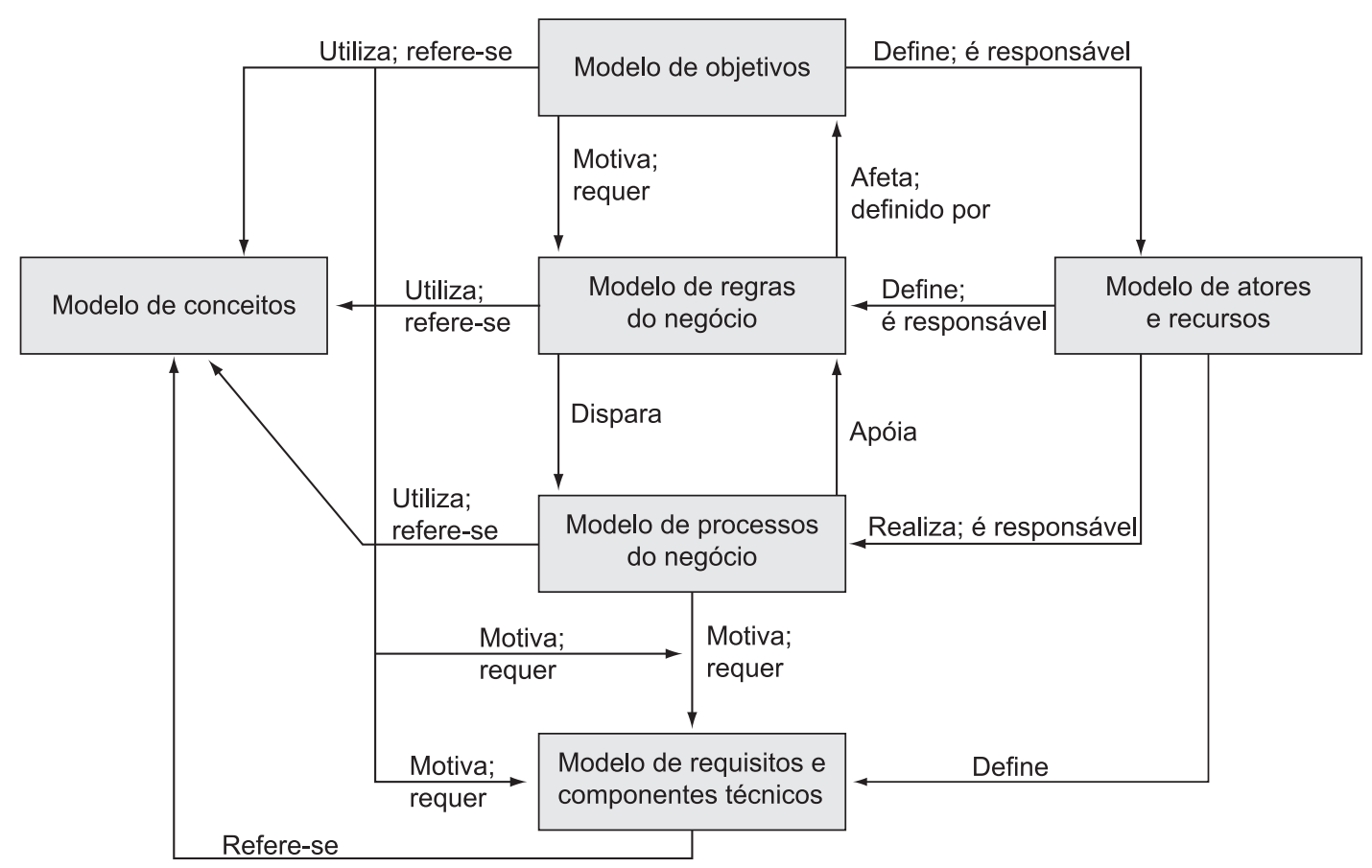

Figura 1. Submodelos do modelo organizacional EKD. Fonte: Bubenko Jr., Brash e Stirna (1998).

projeto de pesquisa maior chamado ICCI, cujo foco baseava-se em uso intensivo de tecnologia de informação. Dentre os projetos que fazem parte do ICCI está o projeto OSMOS, E-COGNOS, E-CONSTRUCT e o E_LEGAL. Todos eles já foram implementados.

Os objetivos do projeto OSMOS, segundo Rezgui, Zarli e Wilson (2000) e Wilson et al. (2001), foram: especificar serviços baseados na Internet para troca de informações entre as equipes virtuais em um cenário dinâmico; e prover ferramentas financeiramente viáveis e de interface amigável para as empresas agirem e participarem destas redes. A modelagem do sistema baseou-se na linguagem de modelagem unificada (UML), e a estrutura de trabalho é dividida em cinco pacotes: capturar requerimentos; definir a arquitetura; implementar a infraestrutura OSMOS; avaliar implementação; disseminar e explorar estrutura; e gerenciar o projeto.

O projeto E-COGNOS, de acordo com Wetherill et al. (2002), especifica e desenvolve um modelo de infraestrutura aberta e ferramentas para o gerenciamento de conhecimento consistente e assimilação de grandes volumes de informação em ambientes cooperativos da construção europeia. A infraestrutura é baseada na web, e a modelagem do sistema utiliza a linguagem de modelagem unificada (UML). Os objetivos do projeto foram: detalhar requerimentos das atividades das companhias, incluindo aspectos organizacionais, contratuais e legais; especificar uma infraestrutura baseada na Internet para criar, classificar, indexar e disseminar conhecimento de forma segura e gerenciar o ambiente que acomoda a complexa informação e interdependências; avaliar e validar o sistema em uso no domínio da construção, e acessar os riscos e benefícios de adotar o padrão proposto.

Similarmente, há o projeto E-CONSTRUCT que, de acordo com Tolman et al. (2001) e Lima, Stephens e Böhms (2003), desenvolve, implementa, demonstra e dissemina uma tecnologia de comunicação de baixo custo, voltada para o comércio eletrônico da indústria de construção europeia.

O projeto E-CONSTRUCT adota a linguagem bcXML (Business Commerce Extensible Markup Language); descreve o que a informação representa. A vantagem está na padronização dos dados elaborados em diferentes softwares. A modelagem do sistema também foi desenvolvida usando a linguagem de modelagem unificada (UML).

O projeto E-LEGAL foi desenvolvido pela Comissão Europeia pela IST (Information Society Technologies). Seu foco, segundo Carter et al. (2001), é definir um cenário para especificar condições legais e contratuais nos processos de negócios, disponibilizar requerimentos de usuários para suporte legal nos projetos da construção; criar "biblioteca" de cláusulas reutilizáveis; configurar contratos e negociação; criar help desk para utilização das ferramentas; e pacote de simulação de negociação para treinamento de aspectos legais na utilização das tecnologias de informação e comunicação entre as empresas componentes das redes virtuais. Entretanto, o projeto E-LEGAL reportou que embora a Internet tenha transformado rapidamente 
a forma de comunicação e troca de informações, as práticas vigentes de contrato têm preservado a forma tradicional, ou seja, no papel.

Os projetos abordados, de forma geral, possuíam benefícios semelhantes, nos quais procuraram: disponibilizar serviços, baseados na Internet, para suprir a necessidade de troca de informação ágil entre fornecedores e parceiros da rede de construção; possibilitar o comércio eletrônico para a rede; e tratar aspectos legais, judiciais e contratuais que envolvem estas redes. Observou-se, também, nos projetos, a adoção da UML, com exceção do projeto E-LEGAL, no qual não houve constatação de utilização de modelagem para os respectivos sistemas de informação, e não de uma metodologia de modelagem. A UML não é uma metodologia, portanto não prescreve explicitamente o procedimento de sua utilização. A sua visualização não é intuitiva, o que se torna um entrave tendo em vista as limitações organizacionais, infraestruturais e culturais do setor de construção civil, conforme Wong, Cheung e Ho (2005).

\section{Método de pesquisa}

A pesquisa deu-se por meio de abordagem de pesquisa bibliográfica, buscando um problema a partir de referências teóricas, de caráter qualitativo, propiciando maior proximidade do pesquisador com os problemas estudados além da análise do contexto pesquisado, e exploratório, disponibilizando correlações entre os objetos de estudo para colaborar com pesquisas posteriores.

A pesquisa analítica, de acordo com Cervo e Bervian (1996) e Yin (2005), evita a duplicidade de conhecimentos e obtém fundamentação teórica para o desenvolvimento do trabalho.

O desenvolvimento da pesquisa dividiu-se em duas etapas. A primeira, com o estudo de sete obras de construção civil, tendo como pré-requisito mínimo de seleção empresas com certificação ISO 9000 e experiência prévia de cooperação entre empresas, além de determinado grau de organização interna reconhecida pelos pares no que tange a melhores práticas gerenciais. Analisaram-se, a seguir, os maiores projetos europeus e americanos na área, incorporando e adequando as melhores práticas de acordo com as características do setor da construção civil no Brasil.

A pesquisa procurou responder às perguntas "como" e "o que deve ser feito" para que as obras de construção civil possam ser configuradas para aproveitar as externalidades potenciais geradas pela rede. As obras investigadas não estavam sob controle dos pesquisadores e discutiram-se eventos contemporâneos cobertos em um período de seis anos (1998 a 2004).

As informações necessárias para a consecução da pesquisa de campo foram obtidas por meio de visitas às empresas e obras envolvidas. Durante as visitas, houve a complementação por meio de entrevistas não estruturadas com profissionais de vários níveis hierárquicos e de documentação pertinente às obras para esclarecer pontos específicos.

A opção por esse tipo de abordagem justifica-se em função das particularidades do setor da construção civil acerca da abordagem da obra sob o recorte analítico de redes. A pesquisa de campo levantou as práticas, confrontando com a teoria, para propor a arquitetura de referência.

Dessa forma, descartou-se um levantamento do tipo "survey" em virtude da falta de profundidade das informações que seriam coletadas. Mesmo com a ausência de uma amostra representativa do setor de construção civil, os casos foram criteriosamente escolhidos.

O Modelo de Componentes e Requisitos técnicos foi elaborado com a utilização da metodologia EKD, no âmbito de NEVES (2007), baseando-se na proposição de Guerrini (2005) referente aos outros submodelos do EKD (objetivos, regras de negócio, processos de negócio e conceitos). Incorporou, também, as melhores práticas dos projetos internacionais de forma a adequarem-se às particularidades do setor da construção civil brasileira, que foram confrontadas com as sete obras analisadas.

\section{Coleta e análise de dados}

A primeira etapa analisou sete obras de construção civil para levantamento dos requisitos de adequação da teoria sobre redes às particularidades das obras de construção. As obras foram denominadas por letras e possuem diferentes agentes de governança, identificados por números. A análise das obras visava identificar as variáveis das relações interorganizacionais. Constatou-se que o motivo principal de descontinuidade é a falta de capacidade que as pequenas e médias empresas do setor possuem para se manterem no mercado. Com a análise da inter-relação sob o ponto de vista do agente de governança, observa-se que existe uma tendência em continuar a relacionar-se com as empresas que atendem a critérios baseados em custos, qualidade do serviço, velocidade e confiabilidade de entrega. E essa continuidade é sustentada por um maior grau de confiança adquirida nessas empresas.

As informações básicas sobre cada obra são descritas no Quadro 1.

O objetivo do processo de formação e gerência de redes de empresas é proporcionar maior poder de competitividade às empresas que delas participam. Para isso, é necessário determinar o ambiente operacional dessa rede para definir os nós. Por meio da caracterização morfológica e tipológica, pode-se determinar os nós atuantes no ambiente operacional de cada obra. Para o processo de formação de redes de 
Quadro 1. Descrição das obras.

\begin{tabular}{|c|c|c|}
\hline Obra & $\begin{array}{l}\text { Agente de } \\
\text { governança }\end{array}$ & Características \\
\hline A & 1 & $\begin{array}{l}\text { Edifício comercial com } 35 \text { andares tipo, } 2 \text { subsolos, restaurante, mezanino, cobertura com } \\
\text { heliponto, etc. Início em fevereiro de } 2003 \text { e término em março de } 2005 \text {. }\end{array}$ \\
\hline B & 1 & $\begin{array}{l}\text { Edifício residencial de } 8 \text { andares tipo com } 4 \text { apartamentos por andar. Feita com recursos } \\
\text { próprios e com período de execução de } 18 \text { meses. Início em novembro de } 2003 \text { e término em } \\
\text { maio de } 2005 \text {. }\end{array}$ \\
\hline $\mathrm{C}$ & 1 & $\begin{array}{l}\text { Edifício residencial com } 20 \text { pavimentos tipo e apartamentos com } 2 \text { e } 3 \text { dormitórios, um } \\
\text { pavimento térreo e } 2 \text { subsolos para garagens. Início em fevereiro de } 2003 \text { e término para } \\
\text { agosto de } 2004 \text {. }\end{array}$ \\
\hline $\mathrm{D}$ & 1 & $\begin{array}{l}\text { Edifício residencial com dois subsolos, } 12 \text { pavimentos tipo, } 48 \text { apartamentos, distribuídos } 4 \\
\text { por andar, cuja construção foi realizada entre } 1 \text { de março de } 2001 \text { e } 30 \text { de agosto de } 2002 \text {. }\end{array}$ \\
\hline $\mathrm{E}$ & 1 & $\begin{array}{l}\text { Edifício residencial com dois subsolos, } 18 \text { pavimentos tipo, } 72 \text { apartamentos, distribuídos } 4 \\
\text { por andar, cuja construção foi realizada entre } 1 \text { de junho de } 2001 \text { e } 30 \text { de junho de } 2003 \text {. }\end{array}$ \\
\hline $\mathrm{F}$ & 1 & $\begin{array}{l}\text { Edifício residencial com dois subsolos, } 16 \text { pavimentos tipo, } 64 \text { apartamentos, distribuídos } 4 \\
\text { por andar, cuja construção foi realizada entre fevereiro de } 2004 \text { e junho de } 2005 \text {. }\end{array}$ \\
\hline G & 2 & $\begin{array}{l}\text { Fórum concluído em 2000. Possui três pavimentos para o público e um técnico. A sua } \\
\text { execução foi dividida em duas fases: a primeira, de } 1997 \text { a 1998, com } 15 \text { meses; e a segunda, } \\
\text { de } 1998 \text { a 2000, com } 18 \text { meses, num total de } 33 \text { meses. }\end{array}$ \\
\hline
\end{tabular}

Fonte: Autores.

empresas, a caracterização morfológica determina as bases estruturais da rede, e a caracterização tipológica é responsável por classificar as relações formadoras dessa rede.

O tipo de relacionamento não tem diferença significativa de uma obra para outra, porém, para as características morfológicas, acontecem muitas mudanças. A caracterização morfológica e tipológica de obra sistematiza o ambiente operacional da obra, identifica as responsabilidades de cada empresa na rede e caracteriza o ambiente operacional ou de tarefas em que aquele conjunto de empresas está atuando.

A caracterização tipológica classifica as parcerias, concernentes ao formalismo, centralização, flexibilidade, direção e fronteiras. A análise das relações interorganizacionais pode determinar características que variam para cada rede de empresas, assim, cada conjunto de relações interorganizacionais pode se comportar e ser classificado de forma diferente.

A caracterização de uma obra como uma rede foi feita utilizando-se os elementos morfológicos (nós (empresas), posições (atividades), ligações (relações) e fluxos (informação e materiais)). Nesse contexto, a morfologia das obras apresentou várias similaridades.

As ligações entre os agentes ocorrem entre os níveis hierárquicos, mas ficam restritas às decisões táticas e operacionais pertinentes à obra. Esse tipo de comportamento revela que as ligações entre os agentes são de caráter colaborativo e não de cooperação, pois as decisões estratégicas dizem respeito somente ao agente de governança. As decisões estratégicas estão relacionadas diretamente com as fases de identificação da oportunidade de negócio e com a fase de configuração da rede. O agente de governança determina os limites da rede e está ligado à responsabilidade legal que o agente tem em relação ao cliente. A formalização dos relacionamentos interorganizacionais ocorre por meio das competências e capacidades complementares, o que caracteriza os fluxos de serviços. Os fluxos de informação têm origem no agente de governança que estabelece e controla o cronograma da obra, medições e pagamentos.

Os nós prestadores de serviço fornecem produtos e serviços para esta obra, porém são simplesmente fornecedores de outras obras. Há flexibilidade na posição que a empresa exerce na rede: ora prestando serviço, ora fornecendo materiais. Como exemplo, as atividades de corte e dobra de aço são realizadas por nós prestadores de serviço; bem como a instalação de kits de portas prontas, colocação de piso de madeira. As empreiteiras estão mais relacionadas com a obra bruta (revestimento externo, regularização de rodapé com impermeabilização e fechamento em alvenaria).

A tipologia das obras estudadas possui as seguintes características em comum: são formalizadas por contratos; as relações entre empresas são realizadas mediante pedidos; possuem um agente de governança, o que caracteriza uma centralização assimétrica; a direção dos relacionamentos é tanto vertical quanto horizontal; há flexibilidade para a substituição de nós sem multas contratuais; possuem uma estrutura virtual na união das empresas por uma oportunidade 
de negócio e, concomitantemente, cada empresa ocupa uma posição bem definida na obra; os nós mantêm um fluxo de informações com o agente de governança, e alguns nós estão ligados por fluxos de materiais; e por fim, observou-se grande rotatividade nas prestadoras de serviço, pois muitas delas têm índice de mortalidade de três anos.

\section{Resultados}

O Modelo de Requisitos e Componentes Técnicos (Figura 2) possui como objetivo principal para o sistema de informação manter uma base de dados com todas as informações relevantes de uma obra (Objetivo S.I 1). Esta meta é apoiada por outros objetivos, os quais: manter dados dos fornecedores (Objetivo S.I .1.1), dos empreiteiros (Objetivo S.I 1.5) e dos contratos realizados (Objetivo S.I 1.6); manter registros atualizados do andamento do ciclo de vida da rede (Objetivo S.I 1.2), do cronograma financeiro (Objetivo S.I 1.3) e o de execução de atividades da obra (Objetivo S.I 1.4). Por fim, manter um relatório de ações inesperadas de cada obra (Objetivo S.I 1.7).

No caso dos fornecedores, dever-se-ia avaliar os critérios competitivos alcançados para cada obra (Req. N.F S.I 2). Para tanto, o sistema armazenará estes dados historicamente (Req. F S.I 4) a fim de serem analisados em futuras consultas, tais como: de qualidade do material fornecido, preço, prazo de entrega, confiabilidade, entre outros aspectos competitivos.

Similarmente, em se tratando dos empreiteiros, dever-se-ia também avaliá-los quanto ao seu comportamento na rede durante a execução da obra (Req. N.F S.I 1). Para tanto, o sistema deve, também, armazenar os dados históricos do desempenho de cada atividade realizada por cada empreiteiro componente da rede que executa cada obra (Req. F S.I 1).

Em se tratando dos cronogramas (financeiro e de execução de atividades da obra) e do andamento do ciclo de vida da rede, faz-se necessário acompanhar todo o ciclo de execução da obra (Req. N.F S.I 5) a fim de obter dados consistentes para futuras avaliações e tomadas de decisões quanto à escolha de parceiros, fornecedores e análise de gastos. Desta forma, o sistema de informação deverá permitir a armazenagem de dados históricos do relatório financeiro de acompanhamento da obra (Req. F S.I 5), além de armazenar dados referentes aos fornecedores e empreiteiros (conforme descrito anteriormente pelos Requisitos Funcionais do Sistema de Informação Req. F S.I 4 e Req. F S.I 1, respectivamente).

Por fim, os contratos também deverão ser armazenados historicamente para não haver risco de perda de dados, bem como para eliminar a quantidade de impressão em papel (Req. N.F S.I 3). Ademais, é relevante haver uma comunicação com um escritório jurídico para assistência legal, judicial e contratual (Req. N.F S.I 4). Para atender a estes requisitos respectivamente, o sistema de informação disponibilizará um mecanismo para autenticação e assinatura digital dos parceiros no contrato (Req. F S.I 2) e integrar-se-á ao escritório jurídico virtual por comunicação online (Req. F. S.I 3). Conforme se nota na Figura 2, o modelo apoia os objetivos, regras e processos definidos para a rede.

\section{Discussão dos resultados}

A discussão dos resultados divide-se em duas partes: a discussão em relação ao referencial teórico, procurando identificar como a literatura contribuiu para a elaboração dos objetivos e regras de negócio; e a discussão relativa às funcionalidades do modelo, no que diz respeito aos objetivos do sistema de informação (Objetivos do S.I.), requisitos funcionais do sistema de informação (R.F.S.I.) e requisitos não funcionais do sistema de informação (R.N.F.S.I.).

\subsection{Discussão dos resultados relativa ao referencial teórico}

O cenário econômico atual requer que as organizações apliquem as melhores práticas de gerenciamento para permanecerem competitivas. Para Belussi e Arcangeli (1998), Chan et al. (2004), Matthews (2004), Rahman e Kumaraswamy (2004) e Jones (2005), as organizações procuram reduzir custos e aumentar o valor agregado dos produtos/serviços. Da mesma forma, afirmam que as organizações devem aprender a lidar com o risco, além de medir a performance durante todas as fases da rede. Essas proposições delimitaram o Objetivo 1 (aumentar ganhos coletivos em critérios competitivos na execução de obras).

A competitividade não deve ser considerada isoladamente ao se estruturar uma rede de cooperação. Para tanto, Belussi e Arcangeli (1998), Camarinha-Matos e Afsarmanesh (1999), Black, Akintoye e Fitzgerald (2000), Tomkins (2001), Travica (2005), Ho (2006) e Ploetner e Ehret (2006) afirmam que se deve relevar: aspectos morfológicos, tipológicos e de governança; ciclo de vida das redes; eliminação de relacionamentos adversos e análise do ponto crítico de se realizar investimentos e contribuições dentro de uma rede. Esse embasamento fortaleceu não somente a delimitação do Objetivo 2 (estruturar a rede de cooperação), como também das Regras 1 (configurar a rede antes de operacionalizar a obra), 2 (operacionalizar a rede durante a execução da obra), 3 (reconfigurar a rede quando for necessário inserir/trocar parceiros), e 4 (dissolver a rede após o término da obra) e dos Processos 1 (configurar a rede), 2 (operacionalizar a rede), 3 (reconfigurar a rede), e 4 (dissolver a rede). 


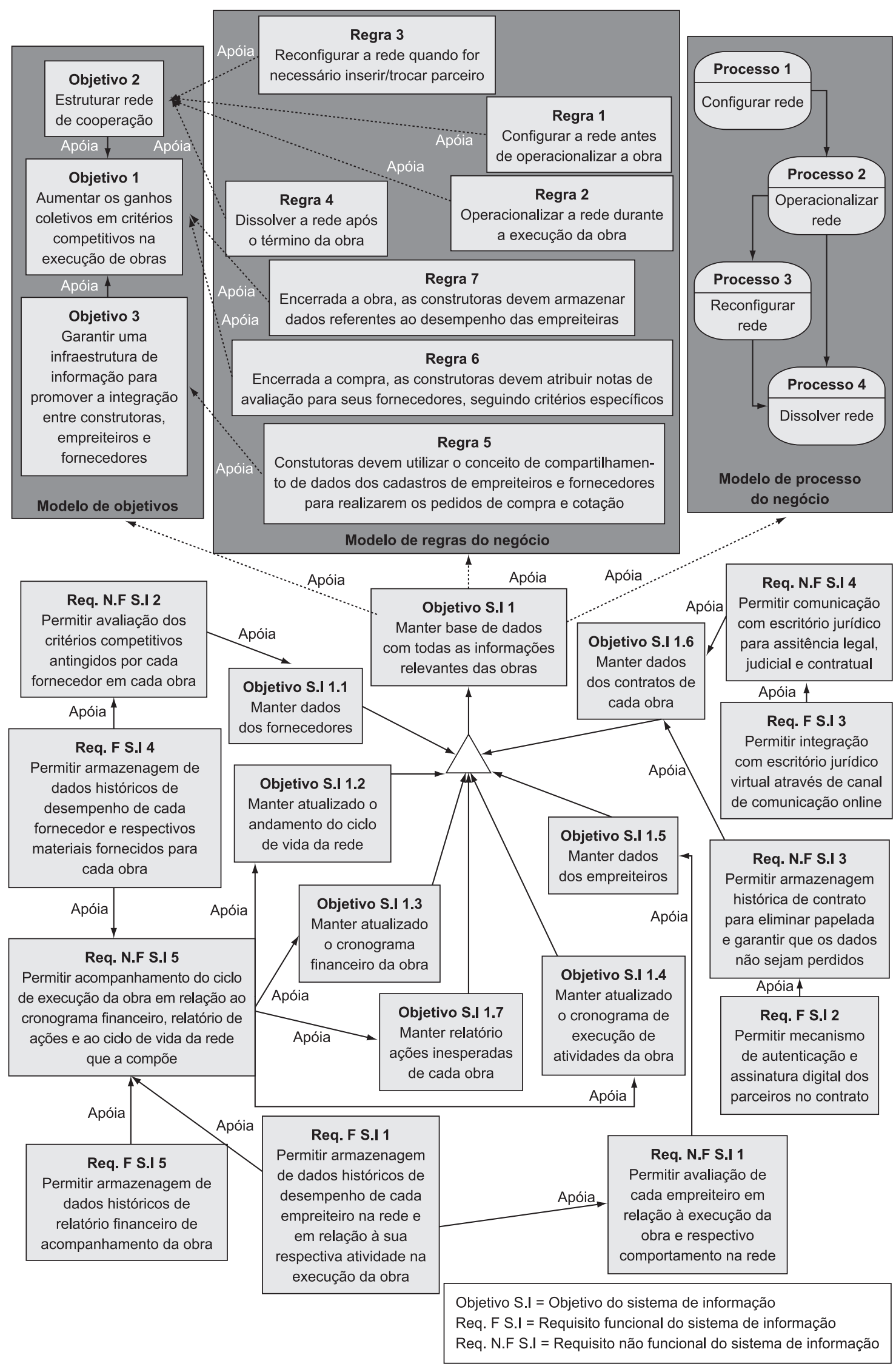

Figura 2. Modelo de requisitos e componentes técnicos. Fonte: Autores. 
Com a estruturação coordenada da rede de cooperação, deve-se projetar o desenvolvimento de uma infraestrutura de informação, uma vez que as redes dependem diretamente do apoio da tecnologia. Para Zarli e Richaud (1999), Tolman et al. (2001), Franke (2003), Lima, Stephens e Böhms (2003) e Breu e Hemingway (2004), a tecnologia de informação deve ser minuciosamente elaborada para dar apoio à necessidade de intensa comunicação e troca de informações. Neste ambiente, cita-se o projeto E-CONSTRUCT, que visa desenvolver, implementar, demonstrar e disseminar uma nova tecnologia de comunicação, voltada para o comércio eletrônico da indústria de construção europeia, para prover uma infraestrutura de comunicação poderosa e de baixo custo. Este direcionamento propiciou a delimitação do Objetivo 3 (garantir uma infraestrutura de informação para promover a integração entre construtoras e fornecedores) e da Regra 5 (construtoras devem utilizar o conceito de compartilhamento de dados dos cadastros de empreiteiros e fornecedores para realizarem os pedidos de compra e cotações).

Importante salientar que o sucesso da rede não é somente garantido pelo apoio de uma infraestrutura de informação adequada. Devem-se adequar, também, os parceiros que compõem a rede. Neste sentido, Black, Akintoye e Fitzgerald (2000), Kelley (2001), Cheng e Li (2002), Glacola e Sheedy (2002), Chan et al. (2004), Kumaraswamy (2005) e Manring e Moore (2006) abordam que o sucesso da rede dependerá, também, da combinação das melhores competências essenciais de cada empresa parceira. Além disto, afirmam que a efetividade da cooperação está diretamente relacionada à intensidade de convicção e motivação de seus parceiros. Entretanto, não se pode deixar de considerar que o ambiente das indústrias de construção civil é vasto e complexo e que, portanto, a informação e comunicação são ferramentas essenciais para melhorar o processo de construção e criar novas oportunidades de negócio.

Segundo Carter et al. (2001) e Wetherill et al. (2002), é inevitável a estruturação de um modelo, uma espécie de mapa padrão com normas comuns. Sabe-se que muitos países europeus têm desenvolvido e disponibilizado implementações das suas regulamentações padrões. Dentro deste cenário, alguns projetos do setor da construção podem ser ressaltados: E-COGNOS (desenvolve infraestrutura flexível para que as organizações assimilem grandes volumes de informações e competências), E-LEGAL (estabelece, também, um help desk para a utilização das ferramentas disponibilizadas). Igualmente relevante, há o aspecto comportamental nas redes virtuais. Para Torkkeli e Tuominem (2002), é imprescindível induzir uma nova filosofia organizacional, uma vez que a informação é vital nos projetos de construção, pois ela especifica o produto resultante e inicia e gerencia as atividades requeridas para tal. Isso propiciou delimitar as
Regras 6 (encerrada a obra, as construtoras devem atribuir notas de avaliação para seus fornecedores, seguindo critérios específicos) e 7 (encerrada a obra, as construtoras devem armazenar dados referentes ao desempenho das empreiteiras).

Por fim, deve-se relevar, conforme Rezgui, Zarli e Wilson (2000), Wilson et al. (2001) e Wong, Cheung e Ho (2005), a difusão efetiva da informação, eliminando barreiras de aprendizado. Além disso, é necessário, ainda, traçar o perfil do membro apto a participar da rede, uma vez que o sucesso não depende somente da tecnologia de informação e comunicação, mas também de fatores críticos comportamentais humanos. Tal afirmação justifica a proposição dos objetivos do sistema de informação, bem como dos requisitos funcionais e não funcionais para constituir um banco de dados que permita o aprendizado contínuo entre os participantes da rede.

\subsection{Discussão dos resultados relativa às funcionalidades}

O Modelo de Requisitos e Componentes Técnicos apoia os modelos de objetivos, regras e processos do negócio para constituir um banco de dados que armazene as informações relevantes das obras (Objetivo S.I 1), relativas a aumentar os ganhos coletivos em critérios competitivos na sua execução; facilitar a estruturação da rede de cooperação e garantir infraestrutura de informação que permita a integração entre os parceiros. Da mesma forma, a manutenção de uma base de dados proporcionará o cumprimento das regras para a armazenagem de dados das obras e avaliação de seus fornecedores (Regras 6 e 7), e ainda compartilhar esses dados com os parceiros da rede (Regra 5). As informações armazenadas servirão de apoio para o cumprimento do ciclo de vida da rede (Regras 1 a 4).

No que tange aos processos do negócio, um banco de dados pode fornecer as informações necessárias para o aprimoramento contínuo do ciclo de vida de uma rede (Processos 1 a 4 ).

Entretanto, o objetivo do sistema de informações de manter a base de dados com todas as informações relevantes das obras apenas será alcançado se o sistema armazenar informações específicas, tais como: dados dos fornecedores e empreiteiros (Objetivos S.I 1.1 e 1.5, respectivamente); andamento do ciclo de vida da rede (Objetivo S.I 1.2); cronograma financeiro e de execução de atividades (Objetivos S.I 1.3 e 1.4, respectivamente); contratos da obra (Objetivo S.I 1.6); e relatório de ações inesperadas (Objetivo S.I 1.7). Cada subobjetivo será apoiado por requisitos funcionais e não funcionais que deverão compor o sistema.

A armazenagem de dados permite o direcionamento da tomada de decisão para ações futuras. A escolha de 
fornecedores e empreiteiros para novas obras dependerá diretamente do desempenho que tiveram nas obras passadas (Requisitos Funcionais S.I: 1, 4 e 5).

Outros ganhos propiciados pelo futuro sistema de informação contemplam a integração em tempo real, por meio de canal de comunicação online, com uma central de assistência jurídica (Requisito Funcional S.I 3), bem como permite a autenticação e assinatura digital dos parceiros no contrato da obra (Requisito Funcional S.I 2).

Contudo, o Modelo de Requisitos e Componentes Técnicos distingue-se pelo fato de ser direcionado a modelar uma situação futura desejada que contemple todas as necessidades apresentadas pelos modelos antecessores componentes da Metodologia EKD.

\section{Conclusão}

O desenvolvimento do Modelo de Componentes e Requisitos Técnicos enseja algumas considerações finais sobre os motivos de adoção do EKD como ferramenta de modelagem organizacional.

Em se tratando das particularidades da indústria da construção civil brasileira relacionadas ao baixo desenvolvimento, resistência às mudanças e cultura conservadora, a adoção da Linguagem de Modelagem Unificada (UML), apesar de bastante utilizada atualmente e adotada nos projetos internacionais analisados, não seria adequada uma vez que sua compreensão não é intuitiva. Indubitavelmente, isto se caracterizaria como um forte obstáculo de aceitação entre os parceiros da rede e, consequentemente, sua adoção falharia.

O trabalho propõe, então, a adoção da metodologia de modelagem do negócio EKD, para facilitar a compreensão do funcionamento do negócio, pelos seus modelos, estimulando o envolvimento e participação ativa de todos os membros do projeto. Desenvolve uma descrição estruturada do negócio para que os analistas da organização possam discutir e determinar mais claramente os objetivos a serem alcançados, as regras necessárias para que estes objetivos se concretizem, os processos que envolvem o negócio e todas as pessoas e recursos envolvidos na organização.

Importante salientar que o método escolhido independe de ferramenta específica para desenvolvimento. Isto torna a proposta viável, uma vez que investimentos drásticos em ferramentas de modelagem de dados para orientação da coordenação da organização não são bem aceitos. Ademais, a modelagem organizacional baseada na metodologia EKD permite que os envolvidos no negócio visualizem, facilmente, as mudanças organizacionais necessárias a serem realizadas a fim de atender às metas estipuladas pelo Modelo de Objetivos.

Os modelos que envolvem a modelagem organizacional, baseada na metodologia EKD, possibilitam a produção do modelo final nomeado Modelo de
Requisitos e Componentes Técnicos, que viabiliza, aos envolvidos no projeto, uma análise detalhada de quais objetivos e requisitos um futuro sistema de informação deverá contemplar a fim de apoiar as proposições dos modelos de negócio anteriores (objetivos, regras e processos do negócio). Ou seja, o modelo proposto direciona-se para a elaboração de um sistema de informação e não mais aspectos organizacionais.

O modelo de Componentes e Requisitos Técnicos da metodologia EKD sistematizou parte da realidade organizacional que convinha para o sistema de informação, de tal forma que o sistema armazene todos os dados relevantes das obras realizadas (cronogramas, relatórios, contratos, desempenho etc.). Isto induzirá um comprometimento efetivo das empresas empreiteiras e fornecedoras, pois suas respectivas inserções em novos projetos dependerão diretamente de seus desempenhos anteriores, os quais passarão a ser armazenados e disponibilizados no sistema para consulta e tomada de decisão precisa das empresas construtoras. A armazenagem de dados relevantes propicia um aprimoramento contínuo da rede, uma vez que se tem a possibilidade de aprender com os erros anteriores.

Manter os dados relevantes de obras anteriores armazenados em um sistema de informação possibilita um direcionamento correto e preciso da tomada de decisão na escolha parceiros da rede para novas obras. Este fator representa um ganho significativo no processo de formação e gerência da rede entre empresas. Ademais, a melhora na comunicação e intensificação da troca de informações entre os parceiros possibilitará a consolidação da rede e, consequentemente, maior flexibilidade nas relações de cooperação entre as empresas.

É importante salientar que na indústria de construção civil brasileira, há a utilização de diferentes plataformas de softwares para a elaboração dos relatórios de acompanhamento das obras. Para tanto, o projeto E-CONSTRUCT sugere a adoção da linguagem bcXML (Business Commerce Extensible Markup Language), a qual descreve a informação, focando no que ela representa de forma mais rápida, segura e muito mais estruturada. Adicionalmente, esta linguagem proporciona, justamente, a vantagem de padronização dos dados elaborados em diferentes softwares. Ou seja, sugere-se a adoção da linguagem bcXML para o futuro sistema de informação.

Cumpre ressaltar que a metodologia EKD permite uma visualização intuitiva dos modelos, com uma progressiva visão crítica do negócio de forma que a cada nova análise, os modelos são, consequentemente, alterados e otimizados. No entanto, essa modelagem organizacional não substitui a engenharia de requisitos de um sistema. 
Como contribuição a futuras pesquisas, propõe-se submeter o modelo à implementação para estruturar e testar um sistema de informação.

\section{Referências}

BALDWIN, N. A.; CARTER, C. T. The use of eletronic information exchange on construction alliance projects. Automation in Construction, v. 8, n. 6, p. 651-662, 1999.

BELUSI, F.; ARCANGELI, F. A typology of networks: flexible and evolutionary firms. Research Policy, v. 27, n. 4, p. 415-428, 1998.

BLACK, C.; AKINTOYE, A.; FITZGERALD, E. An analysis of success factors and benefits of partnering in construction. International Journal of Projects Management, v. 18, n. 6, p. 423- 434, 2000.

BREU, K.; HEMINGWAY, C. J. Making organizations virtual: the hidden cost of distributed teams. Journal of Information Technology, v. 19, n. 3, p. 191-202, 2004.

BUBENKO Jr., J. A.; BRASH, D.; STIRNA, J. EKD user guide. Dpt of Computer and Systems Sciences. Stockholm: Royal Institute of Technology, 1998.

BUHMAN, C. H. Oncoming wave of collaboration. Industrial Engineer, v. 35, n. 8, 2005.

CAMARINHA-MATOS, L. M.; AFSARMANESH, H. Infrastructures for Virtual Enterprises - Network Industrial Enterprises. Norwell: Kluwer Academic Publishers, 1999.

CARTER, C. et al. The E-LEGAL project: specifying legal terms of Contract ICT Environment. Itcon, v. 6, p. 163-174, 2001. Disponível em: <http://www.itcon. org/2001/12 >. Acesso em: 01 dez. 2005.

CERVO, A. L.; BERVIAN, R. A. Metodologia Científica. 4 ed. São Paulo: Makron Books, 1996.

CHAN, A. P. C. et al. Exploring critical success factors for partnering in construction projects. Journal of Construction Engineering and Management, v. 130, n. 2, p. 188-197, 2004.

CHENG, E. W. L.; LI, H. Construction partnering process and associated critical success factors: quantitative investigation. Journal of Management in Engineering, v. 18, n. 4, p. 194-201, 2002.

CHIEN, T. H.; PENG, T. J. Competition and cooperation intensity in a network - A case study in Taiwan simulator industry. Journal of American Academy of Business, v. 7, n. 2, p. 150-155, 2005.

FITZPATRICK, W. M.; DILULLO, S. A. Strategic alliances and management of intellectual properties: the art of the contract. Advanced Management Journal, v. 3, n. 70, 2005.

FRANKE, U. Virtual web organizations revolutionanize business. Information Techonology Newsletter, v. 14, n. 1, 2003.

GUERRINI, F. M. Arquitetura de referência par redes de cooperação entre empresas de construção civil. 2005. Tese (Livre Docência) - Escola de Engenharia de São Carlos, Universidade de São Paulo, São Carlos, 2005.
GILL, J.; BUTLER, R. C. Managing instability in crosscultural alliances. Long Range Planning Journal, v. 36, n. 6, p. 543-563, 2003.

GLACOLA, C. R.; SHEEDY, W. M. Partnering on defense contracts. Journal of Construction Engineering and Management, v. 128, n. 2, p. 127-138, 2002.

HO, C. H. Exchange - based value creation system for network relationships management. The Journal of American of Business, v. 9, n. 1, p. 2002-9, 2006.

HOLMEN, E.; PEDERSEN, A. C.; TORVATN, T. Building relationships for technological innovation. Journal of Business Research, v. 58, n. 9, p. 1240-1250, 2005.

HYDER, A. S.; ERIKSSON, L. T. Success is not enough; the spectacular rise and fall of a strategic alliance between two multinacionals. Industrial Marketing Management, v. 25, n. 9, p. 1-14, 2005.

JONES, O. Managing poblic-provate partnerships: the enactment of a business venture. Technovation, v. 25, v. 6, p. 587-597, 2005.

KELLEY, E. Keys to effective virtual global teams. The academy of management executive, v. 15, n. 2, 2001.

KNOUSE, S. B. Human resource management in virtual organizations. Personnel Psychology, v. 57, n. 2, 2004.

KOSANKE, K.; VERNADAT, F.; ZELM, M. CIMOSA: enterprise engineering and integration. Computers in Industry, v. 40, n. 2-3, p. 83-97, 1999.

LIMA, C.; STEPHENS, J.; BÖHMS, M. The BCXML: supporting ecommerce and knowledge management in the construction industry. Itcon, v. 8, p. 293-308, 2003.

MANRING, S. L.; MOORE, S. B. Creating and managing a virtual inter-organizational learning network for greener production: a conceptual model and case study. Journal of Cleaner Production, v. 14, n. 9-11, p. 891-899, 2006.

MATTHEWS, J. The rise of the virtual company. Supply management, v. 9, n. 15, p. 32-33, 2004.

MERTINS, K.; JOCHEM, R. Architetures, methods and tools for enterprise engineering. International Journal of Production Economics, v. 98, n. 2, p. 179-188, 2005.

NEVES, F. V. F. Modelos de requisitos e componentes técnicos para formação de redes entre empresas da construção civil. 2007. 78 f. Dissertação (Mestrado em Engenharia de Produção) - Escola de Engenharia de São Carlos, Universidade de São Paulo, São Carlos, 2007.

PFOHL, H; BUSE, H. P. Inter-organizational logistics systems in flexible production networks. An organizational capabilities perspective. International Journal of Physical Distribution \& Logistics Management, v. 30, n. 5, p. 388-408, 2000.

PLOETNER, O.; EHRET, M. From relationships to partnerships - new forms of cooperation between buyer and seller. Industrial Marketing Management, v. 35, n. 1, p. 4-9, 2006.

RAHMAN, M. M.; KUMARASWAMY, M. M. Potential for implementing relational contracting and joint risk management. Journal of Management in Engineering, v. 20, n. 4, p. 178-189, 2004.

RAHMAN, M. M.; KUMARASWAMY, M. M. Relational selection for collaborative working arrangements. Journal 
of Construction Engineering and Management, v. 131, n. 10, p. 1087-1097, 2005.

RESE, M. Successful and sustainable business partnerships: how to select the right partners. Industrial Marketing Management, v. 35, n. 1, p. 72-82, 2006.

REZGUI, Y.; ZARLI, A.; WILSON, I. E. Open system for inter-enterprise information management in dinamyc virtual environments. IST-1999-10491. OSMOS, 2000. Disponível em: <http://www.cic.vtt.fi/ projects/osmos/osmos_presentation.pdf $>$. Acesso em: 01 dez. 2005.

RYCROFT, R. W.; KASH, D. E. Self-organizing innovation networks: implications for globalization. Technovation, v. 24, n. 3, p. 187-197, 2004.

SPEKMAN, R. E.; CARRAWAY, R. Making the transition to collaborative buyer-seller relathionship: An emerging framework. Industrial Marketing Management, v. 35, n. 1, p. 10-19, 2006.

STOWELL, C. Real-time collaboration with flair. Communications News, v. 42, n. 3, p. 40-42, 2005.

TOLMAN, F. et al. E-CONSTRUCT: expectations, solutions and results. Itcon, v. 6, p. 175-197, 2001. Disponível em: <http://www.itcon.org/2001/13>. Acesso em: 01 dez. 2005.

TOMKINS, C. Interdependêncies, trust, and information in relationships, alliances and networks. Accounting, Organizations and Society, v. 26, n. 2, p. 161-191. 2001.

TORKKELI, M.; TUOMINEM, M. The contribution of technology selection to core competencies. International Journal of Economics, v. 77, n. 3, p. 271-284, 2002.
TRAVICA, B. Virtual organization and eletronic commerce. Database for Advances in Information Systems, v. 36, n. 3, p. 45-68, 2005.

VERNADAT, F. B. Enterprise modeling and integration: principles and applications. London: Chapman \& Hall, 1996.

WETHERILL, M. et al. Knowledge management for the construction industry: the E-COGNOS project. Itcon, v. 7, p. 183-196, 2002. Disponível em: <http://www. itcon.org/2002/12>. Acesso em: $01 \mathrm{dez} .2005$.

WILLIAMS, T. The purdue enterprise reference architecture and methodology (PERA). Computers in Industry, v. 24, n. 2-3, p. 141-158, 1997.

WILLIAMS, T. Cooperation by design: structure and cooperation in interorganizacional networks. Journal of Business Research, n. 5867, p. 1-9, 2002.

WILSON, I. et al. Enabling the construction virtual enterprise: the OSMOS approach. Itcon, v. 6, p. 83-110, 2001. Disponível em: <http://www.itcon.org/2001/8>. Acesso em: 01 dez. 2005.

WONG, P. S. P.; CHEUNG, S. O.; HO, P. K. M. Contractor as trust in construction partnering - prisioner's dilemma perspective. Journal of Construction and Management, v. 131, n. 10, p. 1045-1053, 2005.

YIN, R. K. Estudo de caso: planejamento e métodos. 3 ed. Porto Alegre: Bookman, 2005.

ZARLI, A.; RICHAUD, O. Requirements and technology integration for it- based business- oriented frameworks in building and construction. Itcon, v. 4, p. 53-75, 1999. 